\title{
Equilibrium and kinetic study on reactive dyes adsorption by palm kernel shell-based activated carbon: in single and binary systems
}

\begin{abstract}
The adsorption of two reactive dyes, Reactive Black 5 and Reactive Red E, onto palm kernel shell-activated carbon (PKSAC) was studied. The effect of the presence of more than one dye in solution on the equilibrium and kinetics of adsorption was investigated. Equilibrium isotherm models were applied to describe the adsorption capacities of single and binary systems. Adsorption of reactive dyes for single system can be represented by the Freundlich and the Redlich-Peterson models. For binary system, the equilibrium was described successfully by the modified extended Freundlich model. Experimental data showed that competitive adsorption for active sites on the carbon surface resulted in a reduction in the overall uptake capacity of the reactive dyes. The rates of adsorption in single system were found to agree well with the pseudosecond-order kinetic model. Finally, the chemical oxygen demand (COD) of the treated reactive dye solutions from single and binary systems showed that a minimum of $4 \mathrm{~g} / \mathrm{L}$ dosage of PKSAC was needed to reduce the COD to an acceptable level according to the Water Quality Guidelines and the Pollutant Fact Sheets Guidelines.
\end{abstract}

Keyword: Adsorption; Dyes; Chemical oxygen demand; Equilibrium; Activated carbon 